\title{
On the Equation $x^{y}=y^{x}$
}

\author{
Y.S. Kupitz and H. Martini
}

Yaakov S. Kupitz wurde 1950 in Israel geboren. Er studierte Mathematik an der Hebrew University in Jerusalem, wo er auch als Schüler von Professor Micha A. Perles auf dem Gebiet der kombinatorischen Geometrie promovierte. Herr Kupitz besuchte zahlreiche diesem Gebiet gewidmete Spezialtagungen und verbrachte einen längeren Forschungsaufenthalt an der Universität in Aarhus (Dänemark). Zur Zeit unterrichtet er Mathematik an der Hebrew University in Jerusalem. Seine Forschungsinteressen ausserhalb der Mathematik beziehen sich auf die Quellen der antiken hebräischen Sprache und das Alte Testament.

Horst Martini wurde 1954 in Grossröhrsdorf bei Dresden geboren. Er studierte Lehramt für Mathematik und Geographie in Dresden, wo er 1984 auch seine Doktorarbeit im Fach Geometrie verteidigte. Nach der Habilitation an der Friedrich-SchillerUniversität in Jena zu einem Thema aus der Konvexgeometrie wurde er 1989 zum o. Hochschuldozenten für Geometrie in Dresden berufen. Gastaufenthalte (auch als Humboldt-Stipendiat) nach der Wende führten ihn u.a. nach Trier und Augsburg, und 1993 nahm er den Ruf auf eine C4-Professur für Geometrie an die Technische Universität Chemnitz an. Die Publikationen von Herrn Martini sind vor allem der klassischen Geometrie und der Konvexgeometrie zuzuordnen. In seiner Freizeit befasst er sich u.a. mit Musik (Klassik, Blues,...) und Geographie (entsprechende Reisen, ausgedehnte Wanderungen).

\section{Introduction}

The equation $x^{y}=y^{x}, x, y>0$, seems to be abandoned in the literature, except for a remote hebrew journal [2] (but see our remarks at the end of this introduction). It is the aim here to give it due treatment.

Die wohlbekannte Kommutativität der Addition und der Multiplikation reeller Zahlen führt auf die Frage nach der Symmetrie der sieben Grundrechenarten Addition, Subtraktion, Multiplikation, Division, Potenzieren, Radizieren, Logarithmieren. Mit anderen Worten stellt sich die Frage: Wie heissen alle Lösungen der Gleichung $x \circ y=y \circ x$, wobei $\circ$ eine der sieben Grundrechenarten bedeutet? Im Falle der Addition und der Multiplikation erfüllen wegen der Kommutativität alle reellen $x, y$ die obige Gleichung, im Falle der Subtraktion ergibt sich nur die triviale Lösung $x=y$, und im Falle der Division erhält man die Lösungen $x= \pm y(x, y \neq 0)$. Im vorliegenden Artikel untersuchen nun die Autoren die entsprechende Problematik im Falle des Potenzierens. Dabei bestimmen sie insbesondere auch alle positiven, ganzzahligen und rationalen Lösungen der Gleichung $x^{y}=y^{x}$. $j k$ 
At first glance it is not clear for which $y>0$ there is a non-trivial solution to the equation $x^{y}=y^{x}, x, y>0$, i.e., a value $x \neq y, x>0$, such that $(x, y)$ is a solution. This is discussed in paragraph 1 , where the set of non-trivial solutions turns out to be punctured at $(x, y)=(e, e)$.

This point turns out to be the accumulation point of the set of rational non-trivial solutions of the equation $x^{y}=y^{x}, x, y>0$ (paragraph 2) and a double (singular) point of the curve whose cartesian equation is $x^{y}=y^{x}$ (paragraph 3 ).

After finishing this paper, [1] came to our notice, in which an interesting historical discussion on the equation $x^{y}=y^{x}, x, y>0$, is given. From this we learn that already L. Euler treated it, and that he discovered the parametric representation (4) given below, from which he drew the rational solutions given here by (5). He also knew the two asymptotes $(x=1$ and $y=1)$ to the curve. In a letter to Goldbach, also Daniel Bernoulli announces that he found the rational solutions. In [4], E. J. Moulton gives a complete discussion of the curve defined by $x^{y}=y^{x}, x, y>0$, including the results of Proposition 1 below (without giving an explicit formulation) and a figure similar to our Figure 2. But the present discussion is superior to Moulton's. With much industry R. C. Archibald (in [1]) finds many works which deal with the equation $x^{y}=y^{x}, x, y>0$, and he gives references to all of them (until 1921). We were not able to find later or recent works dealing with this equation. However, although we did not check the complete literature with the help of Math. Reviews, our impression is that our Theorem 1 (characterization of the rational solutions) is new and that on the whole it gives a complete, clear treatment of the equation $x^{y}=y^{x}$, while other works deal only with special aspects of it.

\section{Non-trivial solutions of $x^{y}=y^{x}$}

In order to avoid later confusion it is preferable to deal with the equation $x^{y}=y^{x}, x, y>$ 0 , in the form

$$
t^{m}=m^{t}, \quad t, m>0,
$$

where $m$ is a "parameter" and $t$ is the "unknown".

Proposition 1 There is a non-trivial solution $t(\neq m)$ to the equation (1) if and only if $1<m \neq e$, and for such an $m$ the solution is unique.

Proof. Put $u(t)=\frac{\ln t}{t}, t>0$, and by taking logarithms in both sides of (1) it is readily seen that (1) is equivalent to

$$
u(t)=u(m), t>0
$$

where $m>0$ is a parameter.

The number of non-trivial solutions to (2) can be expressed in terms of the pre-image set of $u(m)$ under $u$ :

$$
\#(\text { solutions } t \neq m \text { to }(2))=\#\left(u^{-1}(u(m))\right)-1
$$

(where $u^{-1}(s)$ denotes the pre-image set of $s$ under $u$ ). 


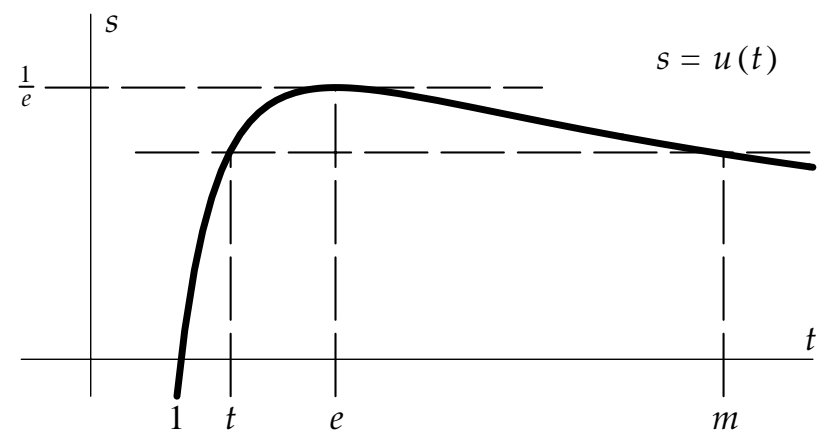

Fig. 1

Since $u^{\prime}(t)=\frac{\frac{1}{t} \cdot t-\ln t}{t^{2}}=\frac{1-\ln t}{t^{2}}, u$ increases on $(0, e]$, attains its maximal value $u(e)=\frac{1}{e}$ at $t=e$, and decreases on $[e, \infty)$. As

$$
\lim u(t)= \begin{cases}0 & \text { for } t \longrightarrow \infty, \\ -\infty & \text { for } t \downarrow 0,\end{cases}
$$

the lines $s=0$ and $t=0$ are horizontal and vertical asymptotes to the graph $s=u(t)$ (Figure 1), respectively.

Thus

$$
\#\left(u^{-1}(s)\right)= \begin{cases}0 & \text { if } \frac{1}{e}<s \\ 1 & \text { if } s \leq 0 \text { or }=\frac{1}{e} \\ 2 & \text { if } 0<s<\frac{1}{e}\end{cases}
$$

and, correspondingly,

$$
\#\left(u^{-1}(u(m))\right)= \begin{cases}0 & \text { if } m \leq 0 \\ 1 & \text { if } 0<m \leq 1 \text { or } m=e \\ 2 & \text { if } 1<m \neq e\end{cases}
$$

Conclusion by (3).

The roles of $m$ and $t$ in Proposition 1 are interchangeable. Hence Proposition 1 applied to $x^{y}=y^{x}$ (where $(m, t) \longrightarrow(x, y)$ ) implies that for $1<x \neq e$ there is a unique $y, 1<y \neq e$, such that $(x, y)$ is a non-trivial solution to $x^{y}=y^{x}, x, y>0$. This defines a surjection $x \longrightarrow \varphi(x)$ from $(1, \infty) \backslash\{e\}$ onto itself. Here the roles of $x, y$ are interchangeable; hence the graph $y=\varphi(x)$ is reflection symmetric around the line $y=x$, i.e., $\varphi(\varphi(x))=x$ (involution).

Inspection of the graph $s=u(t)$ (Figure 1) shows that as $m$ increases on $(1, e)$ the corresponding solution $t \neq m$ of (1) decreases on $(e, \infty)$, and vice versa. Hence $\varphi$ decreases strictly on $(1, \infty) \backslash\{e\}$. Similar considerations show that $\lim _{x \downarrow 1} \varphi(x)=\infty, \lim _{x \rightarrow \infty} \varphi(x)=1$ and $\lim _{x \rightarrow e} \varphi(x)=e$.

The last limit allows to define $\varphi$ continuously at $x=e$ by $\varphi(e)=e$. Since $2^{4}=16=4^{2}$, $\varphi(2)=4$ and $\varphi(4)=2$. The curve $y=\varphi(x)$ is depicted in Figure 2. 


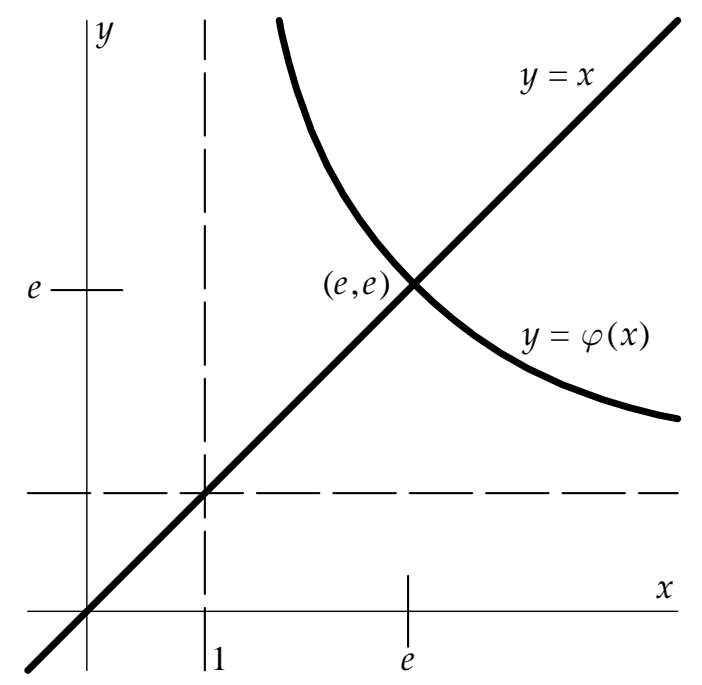

Fig. 2

Proposition 2 The only non-trivial integer solutions to the equation $x^{y}=y^{x}, x, y>0$, are $(2,4)$ and $(4,2)$.

Proof. As 2 is the only integer in $(1, e]$ and $\varphi(2)=4,(2,4)$ is the only integer point on the graph $y=\varphi(x)$ for $1<x \leq e$. By symmetry $(4,2)$ is the only integer point on this graph for $e \leq x<\infty$.

\section{Rational solutions of the equation $x^{y}=y^{x}$}

Let $(x, y)$ be a non-trivial solution of the equation $x^{y}=y^{x}, x, y>0$, and put $y=p x$. Then $p \neq 1, p>0$, and $x^{y}=y^{x}$ can be written in the forms $x^{p x}=(p x)^{x}$ or $\left(x^{x}\right)^{p}=p^{x} \cdot x^{x}$ or $\left(x^{x}\right)^{p-1}=\left(x^{p-1}\right)^{x}=p^{x}$, i.e., we get $x^{p-1}=p$ or, equivalently,

$$
x=p^{\frac{1}{p-1}} .
$$

Put $h=\frac{1}{p-1}, h \in \mathbb{R} \backslash[-1,0]$ (since $p \neq 1, p>0$ ), i.e., $p-1=\frac{1}{h}, p=1+\frac{1}{h}$ and $x=\left(1+\frac{1}{h}\right)^{h}$. As $y=p x, y=\left(1+\frac{1}{h}\right)\left(1+\frac{1}{h}\right)^{h}=\left(1+\frac{1}{h}\right)^{h+1}$. Thus

$$
(x, y)=\left(\left(1+\frac{1}{h}\right)^{h},\left(1+\frac{1}{h}\right)^{h+1}\right),
$$

$h \in \mathbb{R} \backslash[-1,0]$, is a parametric representation of the non-trivial solutions of $x^{y}=$ $y^{x}, x, y>0$. Note that, as $h \longrightarrow \infty,(x, y) \longrightarrow(e, e)$. For $n \in \mathbb{Z} \backslash\{-1,0\}$

$$
(x, y)=\left(\left(1+\frac{1}{n}\right)^{n},\left(1+\frac{1}{n}\right)^{n+1}\right)
$$

is a non-trivial rational solution of the equation $x^{y}=y^{x}, x, y>0$. 
Theorem 1 If $(x, y)$ is a non-trivial rational solution of $x^{y}=y^{x}, x, y>0$, then it is of the form (5) for some $n \in \mathbb{Z} \backslash\{-1,0\}$.

Proof. Since $\left(1-\frac{1}{k+1}\right)^{-(k+1)+1}=\left(\frac{k}{k+1}\right)^{-k}=\left(\frac{k+1}{k}\right)^{k}=\left(1+\frac{1}{k}\right)^{k}$ for $k \in \mathbb{Z} \backslash\{-1,0\}$, it suffices to consider the case $y>x$ and to prove that in this case $(x, y)$ is of the form (5) for some $n \in \mathbb{N}$. So assume that $(x, y)$ is a rational solution of $x^{y}=y^{x}$ with $y>x>0$. Then $p=\frac{y}{x}>1$ is rational and $h=\frac{1}{p-1}$ is a positive rational number. Let $h=\frac{n}{\alpha}$, where $n, \alpha \in \mathbb{N}$ and $(n, \alpha)=1$ (here $(k, l)$ denotes the largest common divisor of $k$ and $l$ ). Then by (4)

$$
x=\left(1+\frac{1}{h}\right)^{h}=\left(1+\frac{\alpha}{n}\right)^{\frac{n}{\alpha}}=\left(\frac{n+\alpha}{n}\right)^{\frac{n}{\alpha}} .
$$

Let $x=\frac{u}{v}$, where $u, v \in \mathbb{N}$ and $(u, v)=1$. Then $\left(\frac{n+\alpha}{n}\right)^{\frac{n}{\alpha}}=x=\frac{u}{v}$, yielding $\left(\frac{n+\alpha}{n}\right)^{n}=$ $\left(\frac{u}{v}\right)^{\alpha}$ and hence

$$
\frac{(n+\alpha)^{n}}{n^{n}}=\frac{u^{\alpha}}{v^{\alpha}}
$$

Since $(n, \alpha)=1,(n+\alpha, n)=1$ and $\left((n+\alpha)^{n}, n^{n}\right)=1$. Similarly, since $(u, v)=$ $1,\left(u^{\alpha}, v^{\alpha}\right)=1$.

It follows that both sides of (6) are the reduced form of the same rational number; hence (by unicity of the reduced form)

$$
n^{n}=v^{\alpha} \text { and }(n+\alpha)^{n}=u^{\alpha} .
$$

Lemma 1 Let $a, b, r, s \in \mathbb{N}$ be such that $a^{b}=r^{s}$. Assume $(b, s)=1$. Then $a=t^{s}$ for some $t \in \mathbb{N}$.

Proof. It suffices to show that if $p$ is a prime factor of $a$ of multiplicity $k$ (i.e., $p^{k} \mid a$ and $\left.p^{k+1} \nmid a\right)$, then $s \mid k$. Since the multiplicity of $p$ in $a^{b}$ is $k b, p^{k b} \mid r^{s}$ and $p^{k b+1} \nmid r^{s}$. Let $m$ be the multiplicity of $p$ in $r$, i.e., $p^{m} \mid r$ and $p^{m+1} \nmid r$. The multiplicity of $p$ in $r^{s}$ is $m s$. Hence $k b=m s$. Since $(b, s)=1, s \mid k$.

Since $(n, \alpha)=1$, Lemma 1 (applied twice in (7)) implies

$$
n=c^{\alpha} \text { and } n+\alpha=d^{\alpha}
$$

for some $c, d \in \mathbb{N}$. Thus

$$
\alpha=d^{\alpha}-c^{\alpha} .
$$

Lemma 2 For $c, d, \alpha \in \mathbb{N}$ with $d>c$ and $\alpha \geq 2$ we have $\alpha<d^{\alpha}-c^{\alpha}$. 
Proof. By induction on $\alpha$.

$\alpha=2$ :

$d>c \Rightarrow d-c \geq 1$ and $d+c \geq 3 \Rightarrow d^{2}-c^{2}=(d-c)(d+c) \geq 1 \cdot 3>2=\alpha$.

Induction step $\alpha \longrightarrow \alpha+1$ :

$$
\begin{aligned}
d^{\alpha+1}-c^{\alpha+1} & =d \cdot d^{\alpha}-c \cdot c^{\alpha}=d^{\alpha}-c^{\alpha}+\left((d-1) d^{\alpha}-(c-1) c^{\alpha}\right) \\
& =\left(d^{\alpha}-c^{\alpha}\right)+\left((d-c) \cdot d^{\alpha}\right)+(c-1)\left(d^{\alpha}-c^{\alpha}\right){ }^{(I)} \alpha+1+0=\alpha+1
\end{aligned}
$$

(Inequality (I) follows from the induction hypothesis.) Thus $d^{\alpha+1}-c^{\alpha+1}>\alpha+1$.

It follows from Lemma 2 that the equation $\alpha=d^{\alpha}-c^{\alpha}$ cannot be satisfied unless $\alpha=1$ (and then $d=c+1)$. Hence $h=\frac{n}{\alpha}=\frac{n}{1}=n$ and

$$
x=\left(1+\frac{1}{h}\right)^{h}=\left(1+\frac{1}{n}\right)^{n}, \quad y=\left(1+\frac{1}{n}\right)^{n+1} \text {. }
$$

This proves Theorem 1.

Corollary 1 The point $P(e, e)$ is the unique accumulation point of the rational points on the graph $y=\varphi(x)$ (= set of rational non-trivial solutions of $\left.x^{y}=y^{x}, x, y>0\right)$.

4 Significance of $P(e, e)$ for the curve defined by the equation $x^{y}=y^{x}$

From the foregoing it follows that the curve defined by $x^{y}=y^{x}, x, y>0$, has two branches:

(i) The line $y=x, x>0$;

(ii) the curve $y=\varphi(x), x>1$.

These two branches intersect at $P(e, e)$. Hence $P$ is a double point of this curve.

This can be checked also by the general theory of singular points. The curve described by $x^{y}=y^{x}, x, y>0$, is a zero level curve of $F(x, y)=x^{y}-y^{x}$, i.e., its equation is $F(x, y)=0$.

Simple calculations show that

$$
\begin{aligned}
& F_{x}=y x^{y-1}-y^{x} \ln y \quad \Rightarrow \quad F_{x}(e, e)=0 \\
& F_{y}=x^{y} \ln x-x y^{x-1} \quad \Rightarrow \quad F_{y}(e, e)=0 .
\end{aligned}
$$

Hence $P$ is a singular point of the curve. The nature of this singularity (isolated/cusp point, double point, or undetermined) is determined by the sign of its discriminant $\triangle_{F}=$ $F_{x x} F_{y y}-F_{x y}^{2}$ at $P$ (see, e.g., [3], p. 84). We have

$$
F_{x x}=y(y-1) x^{y-2}-y^{x}(\ln y)^{2} \Rightarrow F_{x x}(e, e)=e(e-1) e^{e-2}-e^{e} \cdot 1=-e^{e-1} .
$$

Similarly

$$
F_{y y}=x^{y}(\ln x)^{2}-x(x-1) y^{x-2} \Rightarrow F_{y y}(e, e)=e^{e} \cdot 1-e(e-1) e^{e-2}=e^{e-1} .
$$

Hence $F_{x x}(P) F_{y y}(P)=-\left(e^{e-1}\right)^{2}<0$ and

$$
\triangle_{F}(P)=-\left(e^{e-1}\right)^{2}-F_{x y}^{2}(P)<0
$$

Thus $P$ is a double point of the curve defined by $x^{y}=y^{x}, x, y>0$. Figure 2 above depicts this curve and its double point $P(e, e)$. 
Note added in proof: After the acceptance of this paper, the four additional references [5]-[8] below came to our notice (about two of them we were kindly informed by I. Lehmann).

In [8], the solutions of (1) in algebraic numbers are characterized, and [5] contains an approach to the characterization of rational solutions similar to ours. From the didactical point of view, an interesting discussion of (1) is given in [7], and in [6] extensions of (1) are investigated.

\section{References}

[1] R.C. Archibald: Problem notes, No. 9. Amer. Math. Monthly 28 (1921), 141-143.

[2] Yehoshua Bar-Hillel, Y.N. Neman (Eds.): Dapim Lematematika u' lephisika (in Hebrew), Issue No. 6, Jerusalem (March 1943), p. 11, Problem 87 and its Solution in Issue No. 7 (June 1943), p. 10.

[3] R.H. Fowler: The Elementary Differential Geometry of Plane Curves. Cambridge University Press, London, 2nd ed., 1929.

[4] E.J. Moulton: The real function defined by $x^{y}=y^{x}$. Amer. Math. Monthly 23 (1916), $233-237$.

[5] P. Hohler, P. Gebauer: Kann man ohne Rechner entscheiden, ob $e^{\pi}$ oder $\pi^{e}$ grösser ist? Elem. Math. 36 (1981), 131-134.

[6] R.A. Knoebel: Exponentials reiterated. Amer. Math. Monthly 88 (1981), 235-252.

[7] I. Lehmann: Wie symmetrisch sind die sieben Grundrechenarten? Mathematik in der Schule 34 (1996), 344-356.

[8] D. Sato: Algebraic solution of $x^{y}=y^{x}(0<x<y)$. Proc. Amer. Math. Soc. 31 (1972), 316.

Yaakov S. Kupitz

Mathematical Institute

The Hebrew University of Jerusalem

Jerusalem

Israel

Horst Martini

Mathematische Fakultät

Technische Universität Chemnitz

D-09107 Chemnitz

Germany 